\title{
Billion Pierre, Immigrations en Poitou-Charentes. Mémoires de l'invisible
}

Jérémy Guedj

\section{OpenEdition}

Édition électronique

URL : https://journals.openedition.org/remi/6579

DOI : $10.4000 /$ remi.6579

ISSN : $1777-5418$

Éditeur

Université de Poitiers

\section{Édition imprimée}

Date de publication : 1 septembre 2013

Pagination : 166-168

ISBN : 979-10-90426-09-2

ISSN : 0765-0752

Référence électronique

Jérémy Guedj, «Billion Pierre, Immigrations en Poitou-Charentes. Mémoires de l'invisible », Revue européenne des migrations internationales [En ligne], vol. 29 - n³ | 2013, mis en ligne le 01 septembre 2013, consulté le 16 avril 2022. URL : http://journals.openedition.org/remi/6579 ; DOI : https://doi.org/ 10.4000/remi.6579

Ce document a été généré automatiquement le 16 avril 2022.

(c) Université de Poitiers 


\title{
Billion Pierre, Immigrations en Poitou- Charentes. Mémoires de l'invisible
}

\author{
Jérémy Guedj
}

\section{RÉFÉRENCE}

Billion Pierre (2010) Immigrations en Poitou-Charentes. Mémoires de l'invisible, Paris, Autrement, 216 p., ISBN : 978-2-7467-1447-2.

1 À la fin des années 2000, l'Agence nationale pour la cohésion sociale et l'égalité des chances (Acsé) eut l'excellente idée de lancer une grande enquête portant sur l'histoire et la mémoire des immigrations dans les régions françaises. En couvrant l'ensemble du territoire national, l'objectif visait à faire le point des connaissances et à distinguer, sur le temps long, ce que l'on serait tenté d'appeler une France du plein et une France du vide migratoires. L'ouvrage que propose le sociologue Pierre Billion reprend la synthèse historique du rapport consacré à la région Poitou-Charentes et se rattache très nettement au second cas de figure. On ne peut d'ailleurs que se réjouir de la diffusion des résultats de cette enquête ${ }^{1}$, d'autant que, en l'espèce, l'auteur démêle les fils d'une histoire très largement méconnue.

2 D'emblée, Pierre Billion propose une réflexion sur les enjeux historiques et épistémologiques de l'objet qu'il s'apprête à explorer. Il annonce ainsi d'abord la difficulté - et donc, on le comprend, le très grand intérêt - de son entreprise : « Retracer l'histoire des immigrations en région Poitou-Charentes semble, au premier abord, consister à dire l'absence ou presque des immigrés dans la région, à souligner pour le moins leur invisibilité et le silence de leur mémoire » (p.12). Les chiffrent parlent d'eux-mêmes puisqu'au recensement de 1999, la région n'abritait pas plus de $2 \%$ d'immigrés et $1,2 \%$ en 1946. Loin de se laisser décourager par cet obstacle liminaire, l'auteur est parvenu à rassembler une considérable documentation. Second écueil, émanant directement de la commande à l'origine de l'étude : raisonner en termes de région, entité administrative récente et artificielle rapportée à la question de 
l'immigration, pose le problème de l'homogénéité de l'espace exploré. Des ports atlantiques aux campagnes de la Charente, les observations et interprétations ne peuvent que diverger, voire s'opposer. Or cela permet à l'ouvrage de mettre en valeur des lignes de force, des schémas, circuits ou habitudes migratoires peu courants et de se demander si les attitudes des populations locales se révèlent véritablement liées à la densité étrangère.

3 Dès le XIXe siècle, la région accueille, en proportions plus importantes qu'auparavant, des allogènes, ouvriers, travailleurs saisonniers mais aussi réfugiés. Réduits, les flux apparaissent cependant constants au fil des époques et s'accroissent à mesure que l'on entre dans le XXe siècle. Une particularité de la région réside dans le fait que la faiblesse du tissu urbain facilite le contrôle exercé par l'État sur certains étrangers. Ce qui favorise également un phénomène au cœur de l'ouvrage: l'invisibilité des immigrés. Dans l'ensemble, les scansions migratoires de la région s'alignent sur la tendance nationale, mais de manière différée, car l'essor de l'immigration y est plus tardif qu'ailleurs : par exemple, de 1931 à 1936, alors que la population étrangère connaît un affaiblissement dans l'ensemble du pays, elle s'accroît en Poitou-Charentes, phénomène qui persiste encore au recensement de 1946, même si, ne l'oublions pas, il porte en définitive sur des effectifs limités en valeur absolue. La région rejoint le rythme national dans les années 1970.

4 L'échelle retenue permet à l'auteur de scruter très précisément - presque « au ras du sol»- l'évolution de l'immigration. De riches pages sont consacrées à l'attitude des autorités face aux mouvements migratoires qui se déploient au XIXe siècle, en particulier concernant l'encadrement et la surveillance des réfugiés. Plus tard, en 1907, c'est d'ailleurs sur une population de Tsiganes et de voyageurs de Charente-Inférieure que Clemenceau expérimenta le premier carnet anthropométrique. Les mêmes remarquent valent pour des épisodes cruciaux comme les années 1930 ou la guerre naturellement; jusqu'en novembre 1942, la région se trouve d'ailleurs à cheval sur la ligne de démarcation. Sont proposés au lecteur des développements nourris sur le devenir des immigrés, les camps d'internement ou les réactions de la population, même si, sur ces questions, l'on aurait souhaité des comparaisons plus systématiques.

5 Une fois le décor historique solidement planté, Pierre Billion lance l'investigation sur des sujets plus complexes et délicats tels que la mémoire, les mécanismes, s'il en existe, de la xénophobie, l'identité et l'intégration, dont certains jalons ont été posés au cours du récit qui précède. Et ce à partir d'études de cas, à propos desquelles se pose toutefois la question de la représentativité. Les apports s'en révèlent cependant notables. De nombreuses déclarations d'autochtones ont ainsi salué l'activité essentielle des immigrés, notamment dans les tuileries de Roumazières (Charente), mais cela ne doit pas masquer le fait que les étrangers sont avant tout considérés «sur un registre étroitement utilitariste » (p.115). On est frappé de constater, grâce à des exemples précis et circonstanciés, à quel point les représentations apparaissent floues concernant les Algériens à Châtellerault (Vienne), le sort des harkis étant le plus souvent méconnu. L'auteur montre encore qu'à Cerizay (Deux-Sèvres), dans les années 1960 principalement, la société Heuliez accorde sa préférence à des immigrés manifestant une «proximité culturelle et religieuse » (p. 155) avec les Français, ce qui favorise le recrutement des Portugais.

6 Ces exemples témoignent de la variété des aspects abordés dans l'ouvrage, qui témoigne d'un permanent souci de nuance rendant la lecture stimulante. 
Développements généraux, arguments précis et récits de vie, qui donnent de la chair à l'interprétation, alternent. Une interrogation surgit cependant: n'aurait-il pas été profitable d'agrémenter la synthèse commandée par l'Acsé d'un travail archivistique plus poussé, dans un second temps? Proposer un état des connaissances, même remarquablement mené comme c'est le cas ici, conduit le chercheur à être tributaire d'une production existante éclatée, laquelle se justifie par une infinité de paramètres, alors que les cartons d'archives recelant d'insoupçonnées richesses dorment encore. Cela aurait sans doute permis de lier entre eux les divers éclairages proposés et de conférer un caractère plus total, si l'on ose l'expression, à ce récit. Mais, publiée en l'état, cette étude pose un jalon primordial d'une histoire à compléter et, surtout, répond à un impératif civique, celui de connaitre les immigrés. C'est assurément l'un de ses nombreux mérites.

\section{NOTES}

1. Citons notamment, pour d'autres exemples de ce type, Maillard Alain (Dir.) (2009) Les Immigrations en Picardie (XIXe-XXe siècles), Paris, L'Harmattan, 303 p. ou encore Laure Teulières (2010) Histoire des immigrations en Midi-Pyrénées (XIXe-XXe siècles), Toulouse, Loubatières, 176 p.

\section{AUTEURS}

JÉRÉMY GUEDJ

Université de Nice Sophia-Antipolis, Centre de la Méditerranée moderne et contemporaine (CMMC) 